\title{
ANÁLISE BIBLIOMÉTRICA DA PRODUÇÃO CIENTÍFICA INTERNACIONAL SOBRE PEOPLE ANALYTICS
}

\author{
Artur Leonardo Imamura Ferreira da Silva ${ }^{1}$ \\ Eduardo de Camargo Oliva ${ }^{2}$ \\ Edson Keyso de Miranda Kubo ${ }^{3}$
}

\begin{abstract}
RESUMO
O people analytics é uma prática de recursos humanos $(\mathrm{RH})$, possibilitada pela tecnologia da informação, que utiliza análises estatísticas de dados relacionados a processos de $\mathrm{RH}$, criando recortes sobre a situação atual e prevendo situações futuras. $\mathrm{O}$ objetivo desta pesquisa baseou-se no mapeamento das características da produção científica internacional sobre people analytics, a partir de uma amostra de 50 artigos e reviews extraídos base de dados Scopus, sobre as quais foram realizadas análises bibliométricas. Os resultados indicam que (I) a pesquisa sobre people analytics está em estágio inicial e passa por um momento de crescimento, dessa forma, abrem oportunidades de novas correntes de pesquisa; (II) aparentemente não existe predominância exponencial de produção de artigos e reviews de autores, instituições e periódicos prolíficos sobre a temática; (III) as abordagens proeminentes são os aspectos de conceito de people analytics, desenvolvimento operacional e estratégico, o processo de people analytics, a potencialidade e agregação de valor do people analytics, modelos de people analytics. Este estudo contribuiu para evidenciar de forma mais robusta, alargada e atualizada a produção científica do tema people analytics, divulgada na literatura acadêmica internacional, contribuindo para o realce e fomento do conhecimento sobre o citado tema.
\end{abstract}

Palavras-Chave: People Analytics; Human Resources Analytics; Bibliometria.

\section{BIBLIOMETRIC ANALYSIS OF INTERNATIONAL SCIENTIFIC PRODUCTION ON PEOPLE ANALYTICS}

\begin{abstract}
People analytics is human resources $(\mathrm{HR})$ practice enabled by information technology that uses statistical analysis of HR process-related data, creating snippets about the current situation and predicting future situations. The objective of this research was based on the mapping of the characteristics of the international scientific production on people analytics, from a sample of 50 articles and reviews extracted from Scopus database, on which bibliometric analyzes were performed. The results indicate that (I) research on people analytics is at an early stage and is undergoing a moment of growth, thus opening up opportunities for new research streams; (II) apparently there is no exponential predominance of production of articles and reviews by prolific authors, institutions and journals on the subject; (III) the prominent approaches are the concept aspects of people analytics, operational and strategic development, the process of people analytics, the potentiality and value addition of people analytics, models of people analytics. This study contributed to highlight more robustly, broadly and updated the scientific production of the theme people analytics, published in the international academic literature, contributing to the enhancement and fostering of knowledge on the subject.
\end{abstract}

Keywords: People Analytics; Human Resources Analytics; Bibliometric.

'Universidade Municipal de Säo Caetano do Sul, São Caetano Do Sul (Brasil). E-mail: turimamura@yahoo.com.br Drcid id:

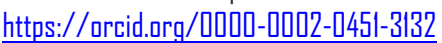

2Universidade Municipal de São Caetano do Sul, São Caetano Do Sul (Brasil). E-mail: eduardo.aliva回uscs.edu.br Drcid id:

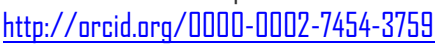

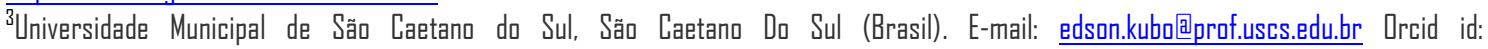

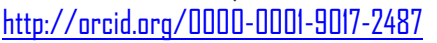




\section{INTRODUÇÃO}

Uma das funções mais importantes dos profissionais de recursos humanos (RH) é avaliar as técnicas de gestão e desenvolvimento de talentos e identificar oportunidades para gerenciar com mais eficácia o capital humano (King, 2016). Como o comportamento humano é muito mais complexo e muito menos previsível do que o de máquinas ou outros ativos tangíveis, a otimização da alocação de capital humano tem sido, historicamente, um empreendimento difícil (Fitz-enz \& Mattox, 2014). Neste sentido, o people analytics, também denominado human resources analytics - HR analytics (Gelbard, Ramon-Gonen, Carmeli, Bittmann \& Talyansky, 2018), tem despontado como um importante investimento que as organizações contemporâneas vêm realizando, pois visa auxiliar e diminuir os erros do estabelecimento de trajetórias no planejamento estratégico de pessoas, assegurando melhores controles e que os colaboradores tenham perspectivas de desenvolvimento e de ascensão profissional mais assertivas, por meio de análises sofisticadas de dados relacionadas aos recursos humanos (Angrave, Charlwood, Kirkpatrick, Lawrence \& Stuart, 2016).

O people analytics, através de sistemas de informação, ferramentas de visualização e análise preditiva, sustentados por perfis de funcionários e dados de desempenho uso, busca auxiliar as organizações a compreender sua força de trabalho como um todo - departamentos, grupos de trabalho e indivíduos - tornando os dados sobre atributos, comportamento e desempenho dos funcionários mais acessíveis, interpretáveis e acionáveis, além de otimizar o recrutamento, retenção, avaliação, promoção, remuneração, volume de negócios e outros aspectos da gestão do capital humano (Tursunbayeva, Di Lauro \& Pagliari, 2018).

Entretanto aplicar o people analytics dentro de uma organização nem sempre é fácil, visto que se cria uma desconfiança inicial dos colaboradores que desconhecem as técnicas de people analytics, suas técnicas e possibilidades de análises podendo gerar interesses individuais e 
organizacionais conflitantes, e que, por conseguinte podem prejudicar a implementação e os resultados positivos (Singer, Storey, Figueira Filho, Zagalsky \& German, 2017).

Pressupondo-se o potencial do people analytics, a presente pesquisa teve como objetivo mapear a produção científica internacional sobre o referido tema. Em vista disso, justifica-se este trabalho à medida que identifica as redes de coautoria, de co-citação e de acoplamento bibliográfico, além dos principais autores e periódicos onde foram publicados os artigos, os núcleos de pesquisa mais relevantes, as respectivas universidades e países, bem como as obras de maior impacto, em linha como o que sugerem Zupic e Cater (2015).

Espera-se que a pesquisa auxilie outros pesquisadores deste tema na condução de suas investigações bem como na identificação de oportunidades de novos caminhos de pesquisa, contribuindo para o avanço do conhecimento científico.

Para isso, o presente artigo encontra-se estruturado em cinco seções, sendo esta introdução à primeira delas. Na segunda seção são abordados os principais conceitos acerca da temática people analytics a fim de facilitar a compreensão e desenvolvimento da pesquisa. Em seguida, são apresentados os procedimentos metodológicos utilizados no trabalho. $\mathrm{Na}$ quarta seção são demonstradas as análises e discussões dos resultados. Por fim, na última seção são expostas as considerações finais, e em sequência as referências utilizadas neste estudo.

\section{FUNDAMENTAÇÃO TEÓRICA}

Esta seção aprofunda os conceitos que serão utilizados como suporte e embasamento científico no decorrer do artigo, abordando no primeiro momento, um panorama geral sobre people analytics, o papel do people analytics na gestão de recursos humanos e os cenários de um projeto de people analytics; e, por fim, o estudo bibliométrico. 


\subsection{People Analytics}

Os gestores dos departamentos de recursos humanos buscam alternativas de melhoria de seus processos de gestão de pessoas, num mercado extremamente dinâmico, marcado pela inovação tecnológica, escassez de talentos e variações de desenvolvimento entre os países. Neste sentido, Boudreau (2017) considera essencial a utilização da tecnologia da informação no RH através do gerenciamento dados relacionados à gestão de pessoas, pois é um fator para alavancar os objetivos da organização.

Fitz-Enz e Mattox II (2014) relatam que com o advento da tecnologia da informação tornou os processos administrativos poderiam se tornar mais eficientes e que os departamentos de $\mathrm{RH}$ não têm agregado valor por não possuírem a expertise em utilizar o modelo estratégico de gerenciamento. O RH dispõe do produto, seja ele software ou um instrumento de pesquisa, contudo eles são meios e não soluções, sendo indispensável uma gestão tecnológica do capital humano baseado em análises.

Nesse contexto emerge a necessidade do uso do people analytics, que segundo Marler e Boudreau (2016) é uma prática de RH possibilitada pela tecnologia da informação que usa recursos descritivos, visuais e análises estatísticas de dados relacionados para realizar análises internas relativas aos processos de $\mathrm{RH}$, que permitam associá-los aos interesses de negócios, e benchmarks econômicos externos para estabelecer o impacto e permitir a tomada de decisão orientada por dados. Para Leonardi e Contractor (2018) people analytics resulta da coleta de dados de fontes distintas que permitem criar um recorte sobre a situação atual e prever situações futuras prováveis na área de gestão de pessoas. Já para Vargas, Yurova, Ruppel, Tworoger e Greenwood. (2018) é o uso de dados, quantitativos e qualitativos métodos de análise e conhecimento de domínio para descobrir insights sobre como as pessoas trabalham em conjunto com o objetivo de melhorar a colaboração.

Conforme Singer, Storey, Figueira Filho, Zagalsky e German. (2017) o people analytics está dividido nas seguintes fases: 
a) Definição de metas: Nesta primeira fase, os gestores do projeto de people analytics definem qual é o assunto de interesse do projeto, o que ele deve descobrir e mais sobre o que é necessário mudar ou melhorar;

b) Coleta de Dados: São escolhidos os dados necessários para atingir o objetivo. Uma coleta estratégica é formulada e os sistemas são configurados para limpeza, agregação, e, de outra forma, proporcionar a manipulação dos dados coletados;

c) Análise e Intervenção: Enquanto ou após os dados serem coletados, o gestor vai executar uma ou várias análises dos dados, implantar uma intervenção - como um sistema de feedback baseado nos dados coletados;

d) Mudança: Dependendo do projeto, uma análise pode fornecer provas ou sugestões para mudanças necessárias em como os colaboradores devem trabalhar juntos, ou como uma intervenção poderia provocar essas mudanças sem ações adicionais e

e) Reflexão: Finalmente, aqueles que implementam o projeto people analytics vão querer refletir se o projeto atingiu o objetivo proposto, 0 porquê funcionou ou possivelmente porque não funcionou. Muitas vezes as novidades em reconfigurações ou sistemas de feedback gera desgaste, tornando-os menos eficazes ao longo do tempo. Nesta fase, é necessária mais coleta de dados para coletar insights sobre os sucessos e fracassos que ocorreram no projeto. Pode ser útil para entender os efeitos de segunda ordem de uma intervenção, incluindo, mas não se limitando as entrevistas qualitativas com os colaboradores afetados.

O people analytics tem muito potencial para melhorar a forma como os colaboradores tomam suas decisões. Ao mesmo tempo, existem vários desafios que precisam ser superados para poder implementar um projeto people analytics bem-sucedido: desconfiança, medo de vigilância, ou a inabilidade para escolha de métricas para análise são apenas alguns exemplos dos problemas que podem surgir (Rasmussen \& Ulrich, 2015; Singer et al., 2017). Neste sentido, os profissionais que implementam um projeto people analytics precisam ser fluentes em diversos métodos de coleta e análise de dados, mas também precisam de expertise na criação de 
intervenções usando insights de interação humano-computador, bem como compreender os processos organizacionais e a ciência de dados, além de aceitar as mudanças para que haja uma compatibilidade dos interesses organizacionais e dos colaboradores, gerando sucesso para todos (Leonardi \& Contractor, 2018).

\subsection{Estudo Bibliométrico}

O estudo bibliométrico, de acordo com o Araújo (2006), consiste numa técnica quantitativa e estatística que tem como enfoque mensurar os índices de produção e difusão do conhecimento científico através da análise quantitativa da informação bibliográfica. Para Macedo, Nova e Almeida (2009) ela auxilia a compreender em que situação a pesquisa de determinada área se encontra.

Para Silva (2004) a bibliometria tem o caráter de analisar a atividade científica através de mapeamento quantitativo das publicações. Rostaing (1997) declara que a bibliometria expressa-se na aplicação dos métodos estatísticos ou matemáticos sobre o conjunto de referências bibliográficas.

Segundo Oliveira et al. (2013), a utilização da pesquisa bibliométrica é uma ferramenta enriquecedora para difusão da produção científica e a seu fim é atingido através da aplicação de técnicas capazes de mensurar a influência de impacto dos pesquisadores e periódicos, permitindo fazer um recorte do perfil e suas inclinações, dessa forma, evidenciando as áreas temáticas. O estudo bibliométrico é o "estudo dos aspectos quantitativos da produção, disseminação e uso da informação registrada" (Macias-Chapula, 1998, p. 134).

Maia (1973) menciona que as características principais do estudo bibliométrico são: elucidar os recursos da comunicação escrita e apontar a evolução de uma determinada área de pesquisa por meio da contagem e análise de suas diferentes abordagens; empregar técnicas estatísticas com os dados correspondentes aos livros e periódicos para elucidar a evolução 
histórica da temática e indicar a aplicação destes conteúdos no cenário nacional e internacional.

Egghe (2005) cita que as leis básicas do estudo bibliométrico derivam de três grandes autores Bradford, Zipf e Lotka. A Tabela 1 apresenta as leis e os seus objetivos.

Tabela 1: Leis básica do estudo bibliométrico

\begin{tabular}{cl}
\hline Leis & \multicolumn{1}{c}{ Objetivos } \\
\hline Lei de Bradford & $\begin{array}{l}\text { Mensurar o nível de relevância dos livros e periódicos de } \\
\text { uma determinada área. }\end{array}$ \\
Lei de Zipf & Medir qual o tema mais abordado nos trabalhos analisados. \\
Lei de Lotka & $\begin{array}{l}\text { Estimar a produtividade e citações de autores por meio de } \\
\text { um modelo de distribuição tamanho-frequência em um } \\
\text { conjunto de estudos, evidenciando aspectos de coautoria }\end{array}$ \\
\hline
\end{tabular}

Fonte: Elaborado a partir de Egghe (2005, pp. 935-945).

$\mathrm{Na}$ próxima seção estão evidenciados os procedimentos metodológicos utilizados para a realização deste estudo.

\section{PROCEDIMENTOS METODOLÓGICOS}

O presente artigo foi desenvolvido a partir de uma pesquisa bibliométrica internacional, com cunho predominante caráter exploratóriodescritivo, objetivando ampliar o conhecimento referente às publicações relacionadas ao tema people analytics, também denominado human resources analytics, destacando as principais características desta produção, permitindo identificar aspectos de destaque no referido campo e potenciais trilhas de pesquisa.

Os dados para realização desta pesquisa foram extraídos da base Scopus na data de 3 de abril de 2019. Segundo (Bar-Ilan, 2008) a referida base é composta de documentos multidisciplinares que indexa as obras mais citadas, dessa forma, proporcionando analisar as citações recebidas, as referências utilizadas e as relacionadas, e principalmente a produção científica sob ótica de rankings, cálculo de índices bibliométrico e a proporção de citações. 
Para tanto, a partir do mecanismo de busca da Scopus, utilizou como palavras-chave "People Analytics" or "Human Resources Analytics" or "HR Analytics" nos campos: título, resumo e palavras-chave, delimitando a busca sem qualquer restrição de período, logo em seguida foi aplicado o filtro limitando a pesquisa aos tipos de documentos "artigos" e "reviews", posteriormente foram pesquisadas as publicações para análise.

A análise dos dados compreendeu dois tipos de análise. Primeiramente, procurou examinar as características gerais das publicações relacionadas a people analytics, tais como: total de publicações, áreas temáticas, ano das publicações, autores, instituições e periódicos; para tanto, tomou-se de suporte o software Microsoft Excel. O segundo esforço foi direcionado à análise bibliométrica, ou seja, mapeamento de redes de coautoria, co-citação, acoplamento bibliográfico, e de co-ocorrência de palavras-chave; estas análises foram realizadas com o suporte do software vOSViewer@, versão 1.6.10.

Segundo Perianes-Rodriguez, Waltman e van Eck (2016) o software VOSViewerC, foi criado por Nees Jan van Eck e Ludo Waltman, e fornece uma interface para visualizar e analisar redes bibliométricas e sociométricas (coautoria, co-citação de autores, citação de países, acoplamento bibliográfico e co-ocorrência de palavras-chave).

O software utiliza a técnica de visualização baseado na distância entre os nós da rede analisada, de modo que a distância entre dois nós indica aproximadamente a intensidade da relação entre eles, sendo tal relação tão maior quanto menor for a distância (Perianes-Rodriguez, Waltman \& van Eck, 2016).

\section{RESULTADOS}

Nesta seção se desenvolve uma discussão dos resultados. Especificamente, são apresentadas, a análise geral das publicações relacionadas à temática people analytics, e por fim, a análise bibliométrica 
(mapeamento de redes de coautoria, co-citação, acoplamento bibliográfico e de co-ocorrência de palavras-chave).

\subsection{Evolução da produção no campo}

Foram encontradas 50 publicações (42 artigos e 8 reviews) em 34 periódicos, e aproximadamente 110 autores e coautores (um número exato de autores não foi computado devido (i) há 3 documentos sem informação de autoria registrada na base, e (ii) à forma como os autores são referenciados nos documentos, podendo haver sutis variações para a designação de um mesmo autor) relacionadas aos temas people analytics, human resources analytics e HR analytics em toda base Scopus, entre os anos de 2012 a 2018, na amostra. A seguir na Figura 1, serão apresentadas as publicações por ano:

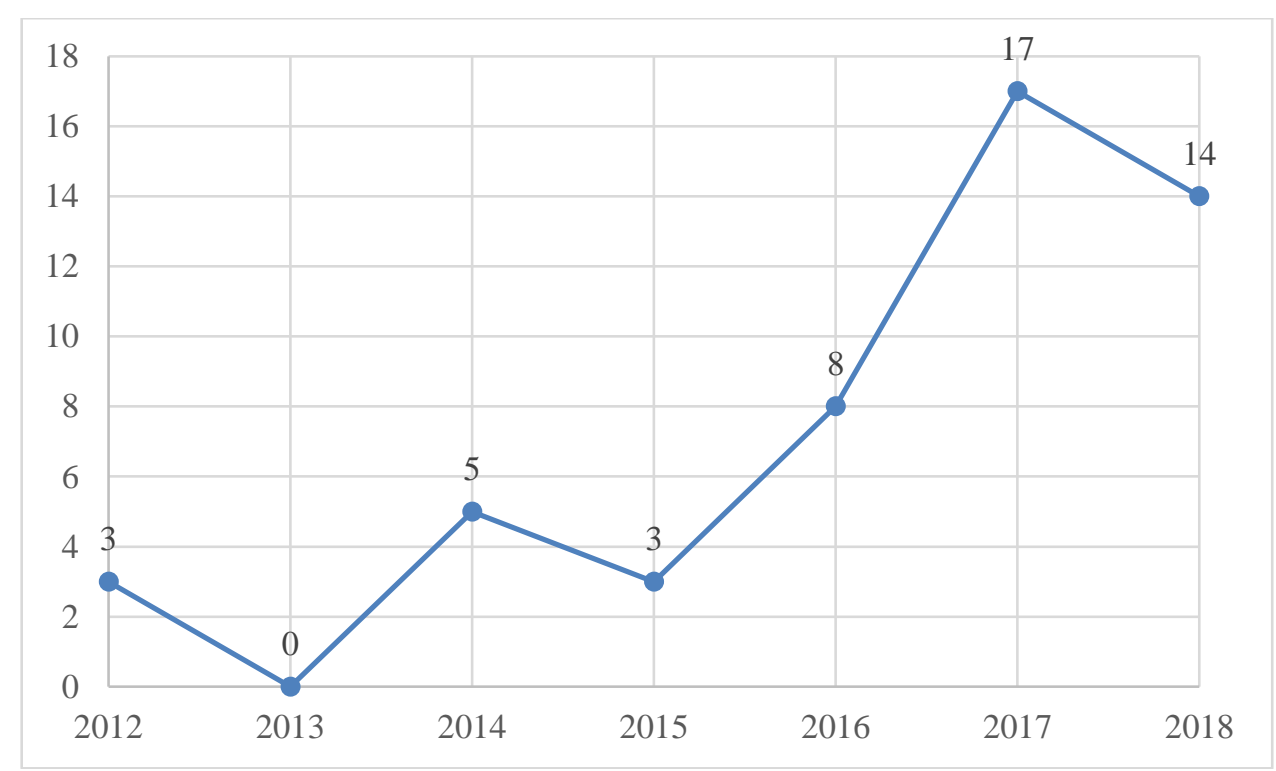

Figura 1: Evolução da produção científica sobre people analytics

Fonte: Elaborado pelos autores (2019). 
Relativamente discreta até 2015, a produção sobre people analytics, conforme a base de dados Scopus, iniciou-se em 2012, com quatro artigos publicados. Um deles de Sinan Aral, Erik Brynjolfsson e Lynn Wu, pois teve maior impacto em termos de citações (63 citações) entre a base de artigos: "Three-way complementarities: Performance pay, human resource analytics, and information technology", publicado no Institute for Operations Research and the Management Sciences (v. 58, n. 5, p. 33-58). Que relata sobre as complementaridades tridimensionais entre $h r$ analytics, tecnologia da informação e remuneração por desempenho.

O ano de 2016 parece representar o momento de inflexão da curva de crescimento na produção sobre o tema, com um aumento de $266 \%$ em relação ao ano anterior. Destaca-se neste ano o artigo "HR and analytics: why $H R$ is set to fail the big data challenge" dos autores David Angrave, Andy Charlwood, Ian Kirkpatrick, Mark Lawrence e Mark Stuart, publicado (2016) Human Resource Management Journal, 26 (1), pp. 1-11. que aborda sobre o potencial transformador da utilização da $h r$ analytics em conjunto do big data para gestão estratégica em recursos humanos.

Quatorze documentos consta na base de dados em edições relativas ao ano de 2018, publicados em 12 diferentes periódicos, o que denota alguma dispersão na produção no campo já naquele ano. A respeito da ligeira queda na quantidade de artigos em 2018, percebe-se que o ano de 2017 parece marcar um período de maturidade da pesquisa, momento em que possivelmente ocorre a estabilização da produção sobre o tema.

No período compreendido entre 2012 e 2018 da amostra, constatouse que o número de publicações aumentou gradativamente ao longo dos anos analisados, evidenciando-se que o crescimento da quantidade de publicações relacionadas ao tema pesquisado teve um crescimento significativo no período, demonstrando a necessidade de estudos que abordam esta temática, propondo meios para auxiliar a gestão de pessoas com o desenvolvimento da coleta dados mais eficientes, eficazes e efetivos que possam predizer o cenário atual e situações futuras de seus colaboradores (Boudreau, 2017; Tursunbayeva, Di Lauro \& Pagliari, 2018). 


\subsection{Composição de autoria}

A análise de composição de autoria das publicações é importante ao permitir tentar compreender, ao longo da história, a densidade com o que os autores têm trabalhado em termos de parcerias de pesquisa com outros coautores. Como esperado, a Figura 2 reflete o crescimento percebido da produção no campo a partir de 2012, para todos os perfis de coautoria (individual, dois autores, três autores, e assim sucessivamente).

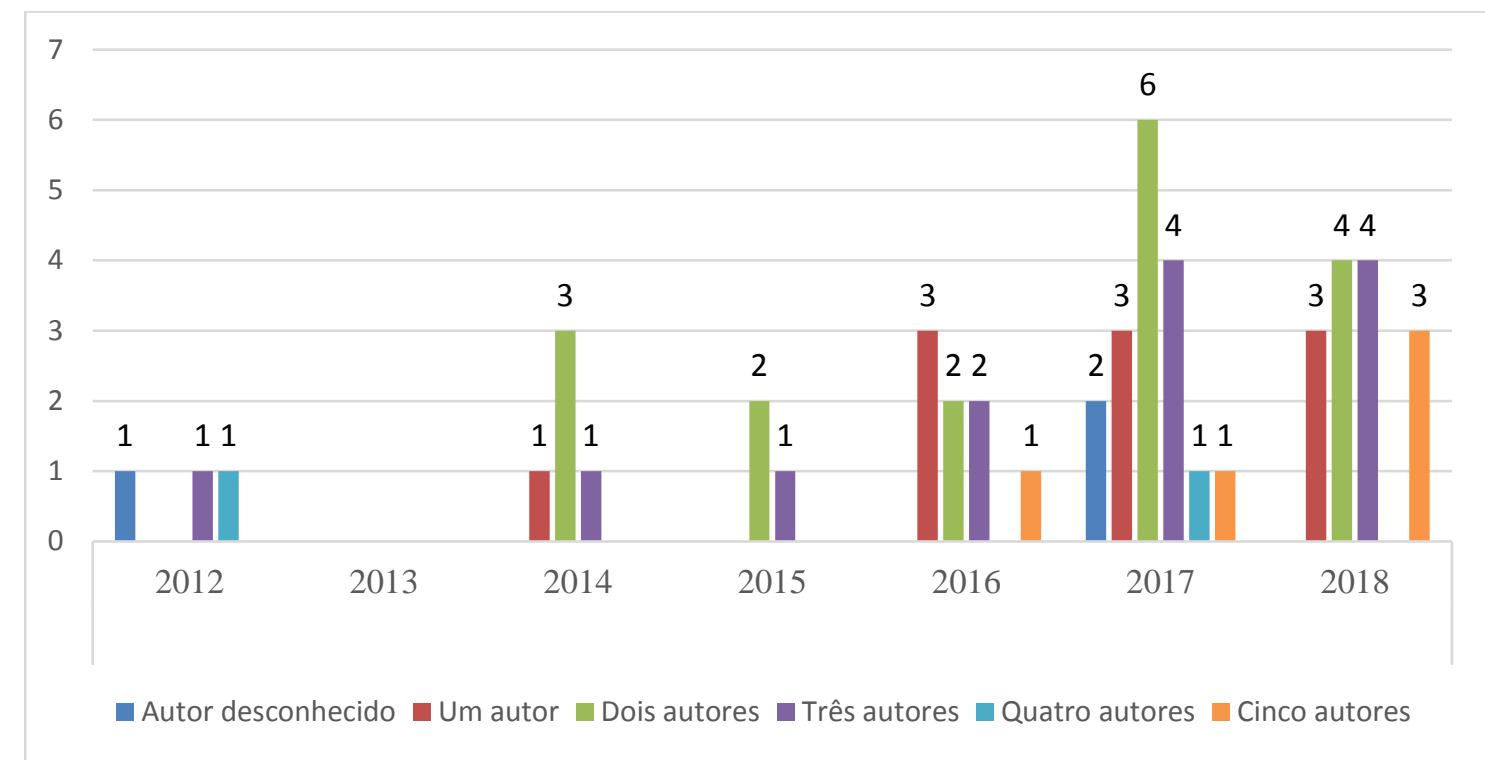

Figura 2: Características da coautoria

Fonte: Elaborada pelos autores (2019).

Considerando todo o período de análise, vê-se $80 \%$ da produção são assinados por entre 1 a 3 autores, sendo mais frequentes, nesta ordem, os trabalhos com dois autores (34\%), três autores (26\%) e de autoria individual (20\%). Trabalhos subscritos por dois autores tiveram seu pico em 2017, com 6 artigos na amostra. Embora tenha apresentado ligeira redução no ano de 2018, ainda é o perfil de coautoria mais frequente juntamente com o perfil de três autores no referido ano. Apesar de pressupor falta de consolidação da produção em termos quantitativos, estas análises sugerem que há um interesse crescente pelo tema hr analytics, ainda que ele esteja 
se manifestando por diversos meios dos campos do conhecimento científico (Ruppel et al., 2018).

Complementando a análise da evolução histórica, alguns resultados relevantes merecem destaque, como autores, instituições e periódicos que publicaram no período de análise.

\subsection{Autores, instituições, periódicos e obras de maior impacto}

A amostra apresentou 110 autores que publicaram sobre os temas People Analytics, Human Resources Analytics e HR Analytics. Observou-se uma diversidade de autores quanto à autoria dos trabalhos, mas uma pequena parcela desses autores - 7 autores: Boudreau, J., Cascio, W., Dexter, F., Fecheyr-Lippens, B., Ledolter, J., Schaninger, B. e Ulrich, D. publicou dois documentos sobre as temáticas e nenhum publicou três ou mais documentos relacionados aos temas.

Outro ponto interessante observado é que 69 instituições da amostra publicaram trabalhos relacionados ao tema People Analytics, Human Resources Analytics e HR Analytics. As instituições que mais se destacaram no que se refere às publicações relacionadas à People Analytics, Human Resources Analytics e HR Analytics foram: a University of Southern California (4 documentos e $1^{\text {a }}$ do rank) e a Royal Dutch Shell (3 documentos e $2^{a}$ do rank), logo em seguida, 6 instituições (SASTRA University, University of Colorado at Denver, Leiden University, University of Iowa, University of Michigan, Ann Arbor e Symbiosis International University) publicaram 2 documentos e as demais instituições 1 documento.

Por fim observou-se que dentro da amostra foram encontrados 34 periódicos que produziram documentos relacionados ao People Analytics, Human Resources Analytics e HR Analytics. Os periódicos que mais produziram sobre a temática foram: Human Resource Management International Digest (5 documentos e $1^{0}$ do rank), Mckinsey Quarterly (3 documentos e $2^{\circ}$ rank) e International Journal Of Engineering And Technology Uae, International Journal Of Human Resource Management, International, Journal Of Information Management e Management Research 
Review (todas com 2 documentos e empatadas em $3^{\circ}$ do rank), os demais periódicos 1 documento.

A partir da Tabela 2 são discutidas as obras presentes na amostra do estudo com maior influência na pesquisa sobre $H R$ analytics, em função do número de citações que receberam de outros documentos publicados em periódicos também indexados à base Scopus. O critério de corte foi o número de citações superior a 12 no período analisado.

Tabela 2: Obras de maior impacto

\begin{tabular}{|c|c|c|c|}
\hline Autor(es) (Ano) & Título & Periódico & $\begin{array}{c}\text { Quantidade de } \\
\text { citações }\end{array}$ \\
\hline $\begin{array}{c}\text { Aral, S., } \\
\text { Brynjolfsson, E., } \\
\text { Wu, L. (2012) }\end{array}$ & $\begin{array}{c}\text { Three-way } \\
\text { complementarities: } \\
\text { Performance pay, } \\
\text { human resource } \\
\text { analytics, and } \\
\text { information } \\
\text { technology }\end{array}$ & $\begin{array}{l}\text { Management } \\
\text { Science }\end{array}$ & 63 \\
\hline $\begin{array}{l}\text { Ulrich, D., } \\
\text { Dulebohn, J.H. } \\
\text { (2015) }\end{array}$ & $\begin{array}{l}\text { Are we there yet? } \\
\text { What's next for HR }\end{array}$ & $\begin{array}{c}\text { Human } \\
\text { Resource } \\
\text { Management } \\
\text { Review }\end{array}$ & 41 \\
\hline $\begin{array}{l}\text { Rasmussen, T., } \\
\text { Ulrich, D. (2015) }\end{array}$ & $\begin{array}{c}\text { Learning from } \\
\text { practice: How HR } \\
\text { analytics avoids } \\
\text { being a } \\
\text { management fad }\end{array}$ & $\begin{array}{c}\text { Organizational } \\
\text { Dynamics }\end{array}$ & 26 \\
\hline $\begin{array}{l}\text { Angrave, D., } \\
\text { Charlwood, A., } \\
\text { Kirkpatrick, I., } \\
\text { Lawrence, M., } \\
\text { Stuart, M. (2016) }\end{array}$ & $\begin{array}{c}H R \text { and analytics: } \\
\text { why } H R \text { is set to fail } \\
\text { the big data } \\
\text { challenge }\end{array}$ & $\begin{array}{c}\text { Human } \\
\text { Resource } \\
\text { Management } \\
\text { Journal }\end{array}$ & 25 \\
\hline $\begin{array}{l}\text { Dexter, F., } \\
\text { Ledolter, J.', } \\
\text { Hindman, B.J. } \\
\text { (2016) }\end{array}$ & $\begin{array}{l}\text { Quantifying the } \\
\text { Diversity and } \\
\text { Similarity of } \\
\text { Surgical Procedures } \\
\text { among Hospitals } \\
\text { and Anesthesia } \\
\text { Providers }\end{array}$ & $\begin{array}{c}\text { Anesthesia } \\
\text { and Analgesia }\end{array}$ & 21 \\
\hline $\begin{array}{c}\text { Marler, J.H., } \\
\text { Boudreau, J.W. } \\
\text { (2017) }\end{array}$ & $\begin{array}{c}\text { An evidence-based } \\
\text { review of } H R \\
\text { Analytics }\end{array}$ & $\begin{array}{c}\text { International } \\
\text { Journal of } \\
\text { Human } \\
\text { Resource } \\
\text { Management }\end{array}$ & 14 \\
\hline $\begin{array}{l}\text { Lismont, J., } \\
\text { Vanthienen, J., } \\
\text { Baesens, B., }\end{array}$ & $\begin{array}{l}\text { Defining analytics } \\
\text { maturity indicators: } \\
\text { A survey approach }\end{array}$ & $\begin{array}{l}\text { International } \\
\text { Journal of } \\
\text { Information }\end{array}$ & 13 \\
\hline
\end{tabular}




\begin{tabular}{|c|c|c|c|}
\hline $\begin{array}{c}\text { Lemahieu, W. } \\
\text { (2017) }\end{array}$ & \multicolumn{3}{|c|}{ Management } \\
\hline $\begin{array}{c}\text { Sinha, V., } \\
\text { Subramanian, } \\
\text { K.S., } \\
\text { Bhattacharya, S., } \\
\text { Chaudhuri, K. } \\
\text { (2012) }\end{array}$ & $\begin{array}{c}\text { The contemporary } \\
\text { framework on social } \\
\text { media analytics as } \\
\text { an emerging tool } \\
\text { for behavior } \\
\text { informatics, HR } \\
\text { analytics and } \\
\text { business process }\end{array}$ & Management & 12 \\
\hline & $\begin{array}{c}\text { Outros (21) - entre } \\
1 \text { e } 11 \text { citações } \\
\text { Outros ( } 21)-0 \\
\text { citações }\end{array}$ & & $\begin{array}{c}50 \\
0\end{array}$ \\
\hline & & Total & 265 \\
\hline
\end{tabular}

Fonte: Elaborado pelos autores (2019)

O trabalho de maior impacto foi o de Aral, Brynjolfsson e Wu (2012) Three-way complementarities: Performance pay, human resource analytics, and information technology, publicado na Management Science (63 citações). É uma obra que estuda três dimensões - tecnologia da informação, remuneração por desempenho e hr analytics - e como elas trabalham em conjunto, produzindo maior produtividade. Em seguida, aparece o artigo de Ulrich e Dulebohn (2015) Are we there yet? What's next for $H R$, publicado na Human Resource Management Review, que aborda sobre o futuro da gestão de recursos humanos e como o $\mathrm{hr}$ analytics pode agregar valor à organização. O terceiro artigo de maior impacto foi publicado na Organizational Dynamics; trata-se da obra de Rasmussen e Ulrich (2015) Learning from practice: How HR analytics avoids being a management fad, que explicita como análise de recursos humanos é apontada como tendo o potencial de trazer grande valor à tomada de decisões dos gerentes gerais e de $\mathrm{RH}$ sobre o capital humano e organizacional, suplementando a intuição e a experiência com evidências.

Outros cinco artigos tiveram mais de 85 citações. São eles, em ordem decrescente de quantidade de citações: Angrave, D., Charlwood, A., Kirkpatrick, I., Lawrence, M., Stuart, M. (2016) HR and analytics: why HR is set to fail the big data challenge Human Resource, Management Journal; Dexter, F., Ledolter, J., Hindman, B.J. (2016) Quantifying the Diversity and 
Similarity of Surgical Procedures among Hospitals and Anesthesia Providers Anesthesia and Analgesia, Anesthesia and Analgesia; Marler, J.H., Boudreau, J.W. (2017) An evidence-based review of HR Analytics International, Journal of Human Resource Management; Lismont, J., Vanthienen, J., Baesens, B., Lemahieu, W. (2017) Defining analytics maturity indicators: A survey approach; International Journal of Information Management e Sinha, V., Subramanian, K.S., Bhattacharya, S., Chaudhuri, K. (2012) The contemporary framework on social media analytics as an emerging tool for behavior informatics, HR analytics and business process Management.

Destes oito artigos de maior impacto, nota-se que o pesquisador Dave Ulrich é autor e coautor em dois deles, além do autor com maior número de citações (67 citações), demonstrando sua relevância na pesquisa sobre people analytics. Ao todo, a tabela apresenta oito artigos (16\% da amostra) com o maior volume de citações (mais de 12). Estas obras correspondem a $81,13 \%$ do total de citações presentes na base analisada, 0 que denota sua relevância e seu impacto para a pesquisa na área. Importante destacar ainda que ainda 21 artigos, ou seja, quase $42 \%$ da amostra ainda não receberam nenhuma citação.

\subsection{Rede de coautoria}

A análise de redes de coautoria (van Eck \& Waltman, 2014) é uma técnica de análise para identificar como autores, instituições ou países se relacionam conforme a quantidade de estudos que realizam e publicam conjuntamente. Para identificar os aspectos de colaboração entre os autores mais relevantes utilizou-se o método de força de associação pelo software VOSViewer $@$ que normaliza a força das ligações entre os itens, a qual será maior, tanto maior for a frequência de documentos produzido por um conjunto de autores (van Eck \& Waltman, 2014). A Figura 3 apresenta as principais redes de coautoria mapeadas. 

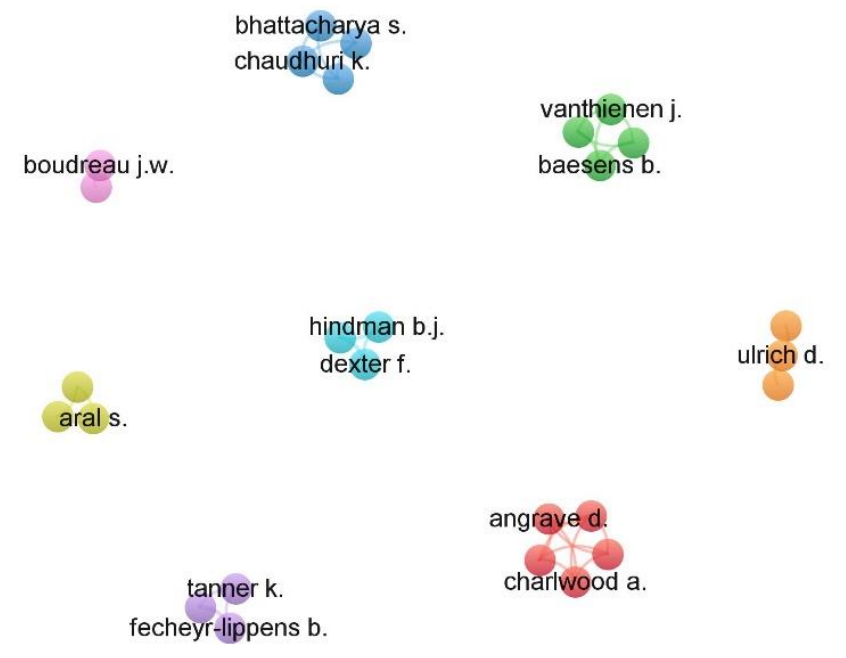

VOSviewer

boudreau j.

\section{Figura 3: Redes de coautoria}

Fonte: Elaborada pelos autores (2019)

Foram considerados apenas os autores com pelo menos 1 documento na amostra, e pelo menos 5 citações a suas respectivas obras. Tal restrição produziu uma rede com 29 nós (autores), distribuído em 9 clusters de colaboração de pesquisa, denotando a dispersão da pesquisa no campo.

O tamanho dos círculos indica a quantidade de publicações de cada autor na amostra. Os clusters com maiores números de nós, portanto, com mais autores colaborando entre si aparecem em destaque na Figura 3 (cluster 1 - vermelho, cluster 2 - verde e cluster 3 - azul escuro). O cluster 1 (vermelho) é constituído por cinco autores Angrave D., Charlwood A., Kirkpatrick I., Lawrence M. e Stuart M., sendo que eles possuem o mesmo número de links com os demais deles, 4 links e 4 links strenght cada autor. O cluster 2 (verde) é constituído por 4 autores e não apresenta centralidade entre seus autores, Baesens B., Lemahieu W., Lismont J. e Vanthienen J., pois eles também possuem o mesmo número de links com os demais deles, 3 links e 3 links strenght cada autor. O cluster 3 (azul escuro) apresenta as mesmas características do cluster 2, possuindo 4 autores Bhattacharya S., 
Chaudhuri K., Sinha V. e Subramanian K. K. e 3 links e 3 links strenght cada autor. Quatro outros clusters (cluster 4 - amarelo, cluster 5 - violeta, cluster 6 - azul claro e cluster 7 - laranja) são formados por 3 autores e dois clusters (cluster 8 - marrom e cluster 9 - rosa) de dois autores, resultado que corrobora a dispersão da produção no campo de People Analytics, Human Resources Analytics e HR Analytics. (Boudreau, 2017; Tursunbayeva et al., 2018).

\subsection{Redes de co-citação de autores}

A avaliação de co-citação permite determinar a regularidade com que 2 itens (usualmente papers ou autores) da bibliografia prévia são citados juntos por algum item da bibliografia mais atual (Small, 1973). Em síntese, 2 autores (ou duas publicações) são ditos(as) co-citados(as) quando há um (a) terceiro (a) que os(as) cita conjuntamente. Dessa forma, quanto maior o número de documentos em que 2 autores (ou duas publicações) são co-citados(as), mais forte será a correlação de co-citação entre esses(as) (van Eck \& Waltman, 2014). A Figura 4 mostra a rede de relacionamentos de co-citação de autores. O critério de corte foi o número mínimo de 5 citações, o que levou a uma rede de co-citação de 50 autores (nós).

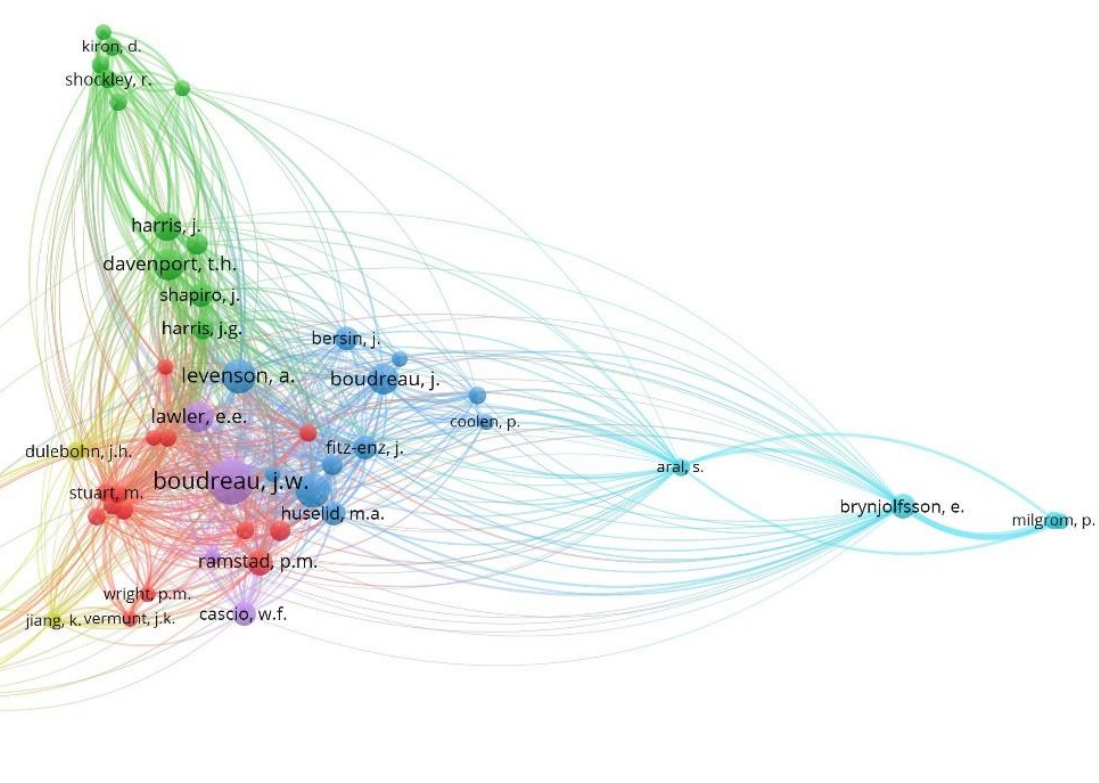

Figura 4: Redes de co-citação de autores 
Fonte: Elaborada pelos autores (2019)

Nesta avaliação, a dimensão de cada nó da rede indica o volume de citações que o próprio autor recebeu, e não mais o número de artigos publicados, como na avaliação de coautoria. Quanto mais próximos 2 nós se encontram, mais forte é a correlação de co-citação entre eles; ou seja: maior é o número co-citações, ou de "terceiros autores" que citaram os dois conjuntamente.

A rede de co-citações é distribuída em seis clusters. O primeiro cluster (vermelho) é constituído por 14 autores, Ajzen I., Angrave D., Charlwood A., Craig E., Kirkpatrick I, Lawerence M., Lepak D. P., Light D. A., Marler J. H., Paauwe J., Ramstad P. M., Stuart M., Vermunt J. K. e Wright P. M. Tomando por referência os estudos empreendidos por estes pesquisadores, observa-se o cluster 1 concentra principalmente autores que investigam a perspectiva que as organizações devem compreender em conjunto o procedimento operacional de people analytics quanto ao desenvolvimento estratégico de metodologias de recursos humanos e a consolidação do conceito de HR analytics.

O segundo cluster (verde) é composto por 11 pesquisadores, com destaque para os seguintes mais citados: Davenport T., Harris J. e Shapiro J.. Estes autores tradicionalmente têm pesquisado os processos de people analytics com um diferencial para tomada de decisões mais conscientes.

O terceiro cluster (azul escuro) da rede de co-citações é formado por 11 autores. Os mais relevantes deles são Boudreau J., Levenson A. e Ulrich D.. As pesquisas realizadas por estes autores, tomadas como base para caracterização deste grupo, são predominantemente direcionadas sobre a potencialidade e agregação de valor do $h r$ analytics, estudo de modelos de people analytics, relação entre processos de recursos humanos e a incorporação de tecnologias.

Já o quarto cluster (amarelo) é formado por 6 autores - Dulebohn J. H., Eisenberger R., Jiang K., Lee T. W., Mitchell T. R. e Porter L. W. O grupo tem como caracterização direcionada ao suporte da tecnologia da informação nos processos de recurso humanos. 
O quinto cluster (violeta) é composto por 4 pesquisadores, entre os mais relevantes Becker B. E., Cascio W. F. e Lawler E. E. Os estudos realizados por estes pesquisadores focam sobre a influência da gestão de recursos humanos no desempenho organizacional com auxílio do people analytics.

O sexto cluster (azul claro) é constituído por 4 autores, sendo Aral S., Brynjolfsson E, Hitt. L. M. e Milgrom P. O último grupo tem como aspectos o foco à necessidade de mão de obra qualificada para manusear a tecnologia da informação em people analytics.

\subsection{Redes de citação por países}

A análise de rede de citação por países mensura o impacto e a visibilidade de países dentro de uma comunidade científica, demostrando os países que cooperam em determinados campos da ciência e sua relevância (Moraes, Furtado \& Tomaél, 2015).

A amostra apresentou documentos de 16 países diferentes: Estados Unidos (20 documentos), Índia (11 documentos), Holanda (5 documentos), Reino Unido (5 documentos), Bélgica (3 documentos), China (2 documentos), Dinamarca (2 documentos), Austrália (1 documento), Brasil (1 documento), Alemanha (1 documento), Israel (1 documento), Itália (1 documento), Japão (1 documento), Filipinas (1 documento), África do Sul (1 documento) e Suíça (1 documento), além de contar com 4 documentos que não foi possível a identificação do país. A Figura 5 mostra a rede de relacionamentos de citação por países. O critério de corte foi o mínimo de 1 documento por país e o mínimo de 2 citação por países, o que levou a uma rede de citação de países de 7 países. 


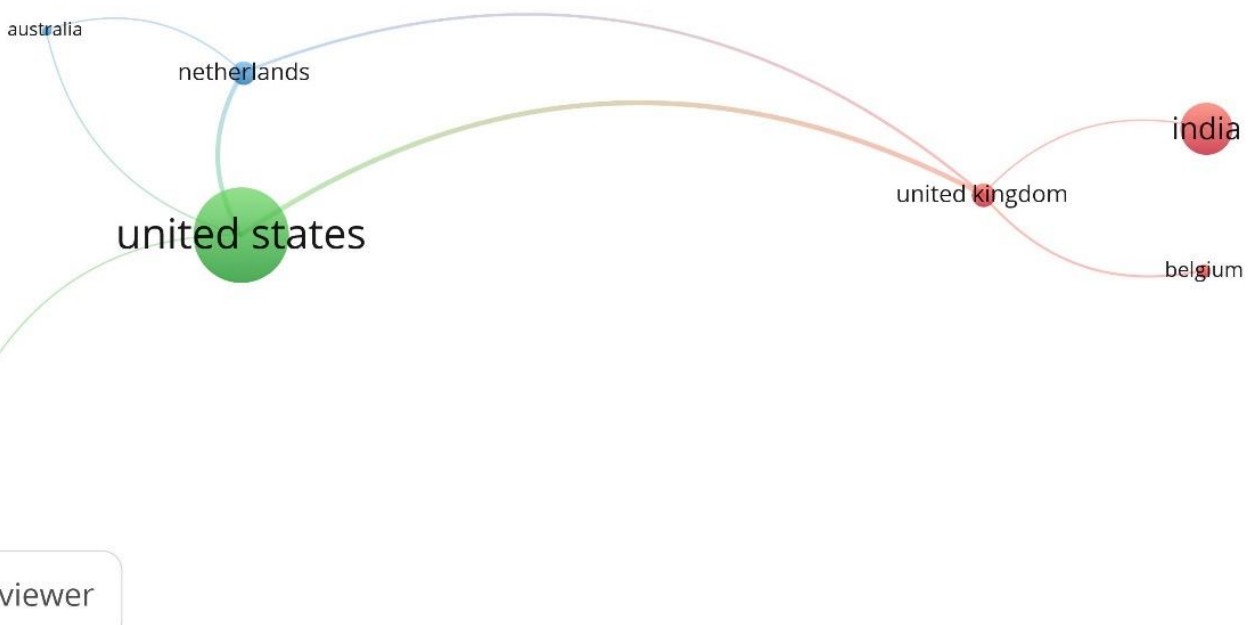

Figura 5: Redes de citação por países

Fonte: Elaborada pelos autores (2019)

A rede de citação é distribuída em três clusters. O primeiro cluster é constituído pelos seguintes países: Bélgica, Índia e Reino Unido. O segundo cluster é formado por Israel e Estados Unidos. Enquanto o terceiro cluster é formado Austrália e Holanda. Resultado que corrobora a concentração da produção no campo e as principais redes de citação por países de People Analytics, Human Resources Analytics e HR Analytics.

\subsection{Redes de acoplamento bibliográfico}

O acoplamento bibliográfico pode ser percebido como o "oposto" da co-citação. Uma referência que é citada por 2 artigos é considerada um elemento de acoplamento entre estas (Kessler, 1963). Isso implica que 2 autores (ou duas publicações) são considerados(as) acoplados(as) bibliograficamente cada vez que houver um (a) terceiro (a) autor (publicação) que é citado (a) ao mesmo tempo pelos(as) primeiros(as). Em síntese, quanto maior o número de referências que 2 autores ou 2 artigos compartilham, maior a intensidade do acoplamento bibliográfico entre eles 
(van Eck \& Waltman, 2014). O pressuposto aqui é o de que publicações "acopladas" tratam temáticas conceitualmente próximas, indicando núcleos referenciais de pesquisas e fomentando a execução de novas pesquisas no campo.

As redes de acoplamento bibliográfico entre os autores da amostra são apresentadas na Figura 6.

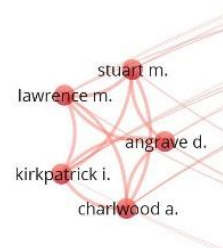

\section{象 Vosviewer}

\section{Figura 6: Redes de acoplamento bibliográfico}

Fonte: Elaborada pelos autores (2019)

Como critérios de corte, restringiu-se a rede a autores com pelo menos um documento na base de dados e com no mínimo doze citações, o que resultou em uma rede constituída por 10 autores, dispostos em 3 clusters. Cada pesquisador presente em um cluster tende a citar os mesmos autores que os demais pesquisadores deste mesmo cluster, pelo que se considera a forte relação de acoplamento bibliográfico.

No cluster 1 reuniu 5 pesquisadores, Angrave D. Charlwood A. Kirkpatrick I. Lawerence $M$ e Stuart $M$. No cluster 2, formado por três autores, Dulebohn J. H., Rasmussen T. e Ulrich D.. Por último, o cluster 3 agrupou dois autores, Boudreau J. W. e Marler J. H. Em comum entre estes principais autores dos clusters 1,2 e 3, o fato de pertencer a instituições dos EUA e de outras europeias, localizadas no Reino Unido e Holanda. 
Como evidenciado na avaliação das redes de co-citação, as redes de acoplamento bibliográfico entre os documentos da amostra parecem evidenciar a presença de núcleos de estudo definidos, com destaque para os autores com afiliações nas instituições americanas, ainda que isto similarmente possa ser resultante da influência da correlação de coautoria entre os autores de cada cluster.

\subsection{Redes de co-ocorrências de palavras-chave}

A correlação de co-ocorrência entre duas palavras-chave é estabelecida pelo número de papers em uma base de documentos em que uma e outra ocorrem conjuntamente, seja no título, no resumo ou na listagem de palavras-chave (van Eck \& Waltman, 2014). Ao ver essas redes, é possível mapear possíveis temáticas de estudo sobre people analytic. A dimensão do nó indica a frequência de ocorrência de uma palavra-chave, e a correlação entre os nós é tão mais forte quanto maior a proximidade entre eles. A Figura 7 apresenta as redes de co-ocorrência de palavras-chave para os 50 documentos da amostra.

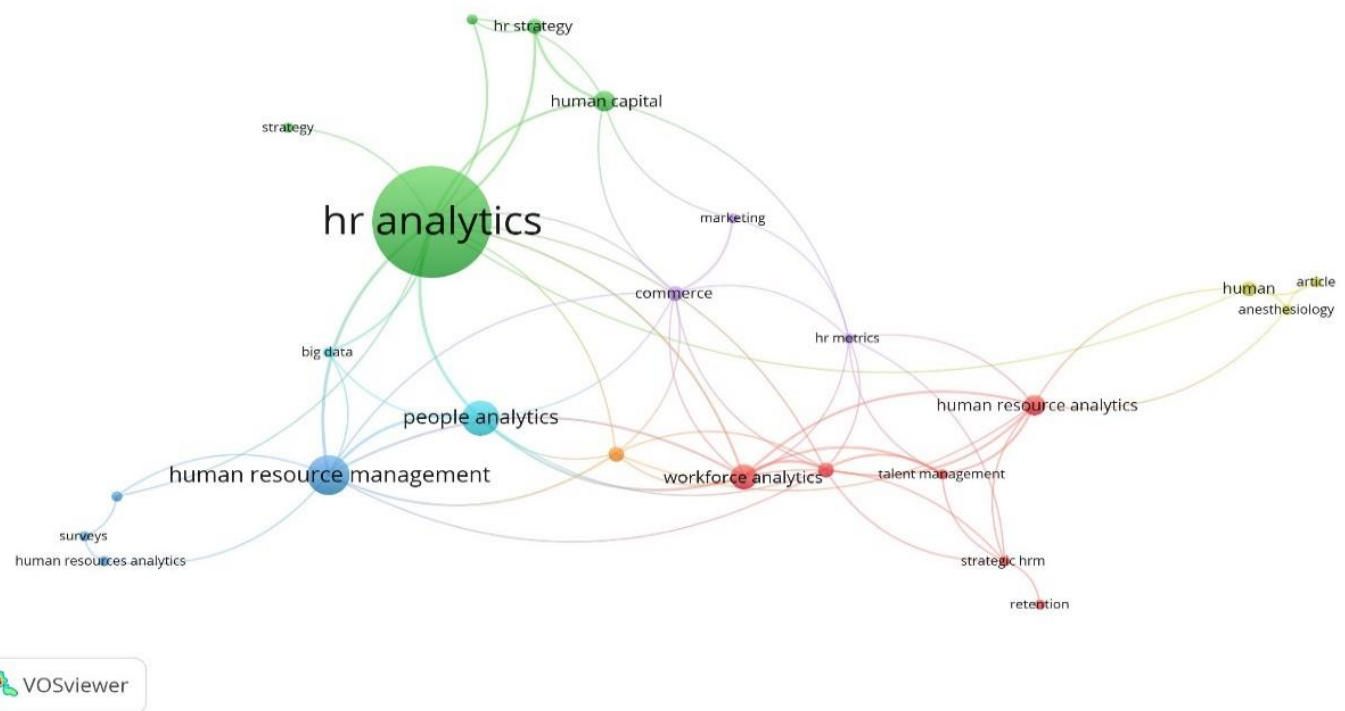

Figura 7: Redes de co-ocorrências de palavras-chave

Fonte: Elaborada pelos autores (2019). 
Para facilitar a visualização, a formação da rede foi restrita as palavras-chaves com duas ou mais ocorrências, o que resultou em 24 nós, organizados em sete clusters. Estas são, portanto, as palavras de maior frequência e que, segundo a Lei de Zipf, determinam a temática central de um corpo de documentos.

No cluster 1 (vermelho), contendo seis nós, no cluster 2 (verde), contendo cinco nós, no cluster 3 (azul), contendo quatro nós, no cluster 4 (amarelo), contendo três nós, no cluster 5 (violeta), contendo três nós, no cluster 6 (azul claro), contendo dois nós e no cluster 7 (laranja), contendo um nó. Nota-se que no cluster 1 (vermelho) concentra as palavras-chave hris (human resource information system), human resource analytics, retention, strategic hrm (human resource management), talent management e workforce analytics. Este conjunto sugere pesquisas que abordam as relações entre o people analytics e a gestão de talentos, à luz dos processos de retenção de talentos como estratégia em recursos humanos.

Entre os cinco nós agrupados no cluster 2 (verde), a própria expressão hr analytics, change management, hr strategy, human capital e strategy. Tais ocorrências permitem concluir como estudos estão considerando o people analytics como uma mudança de gestão e sendo necessário para estratégia e agregando valor para os recursos humanos.

O terceiro cluster (azul) reuniu quatro palavras-chave, humam resource management, human resources analytics, predictive analytics e surveys. A linha de pesquisa que tais palavras sugerem como as pesquisas estão sendo desenvolvidas para tentar predizer os comportamentos humanos na gestão de recursos humanos.

O quatro cluster (amarelo) reuniu anesthesiology, article e human. Este conjunto tenta compreender o comportamento humano e profissional de profissionais da área de saúde através de métricas utilizadas em $h r$ analytics.

O quinto cluster (violeta) reuniu commerce, hr metrics e marketing. O grupo tenta compreender o cenário do comércio e marketing, além de 
como as métricas de recursos humanos podem impulsionar a organização, principalmente nestes setores.

O sexto cluster (azul claro) reuniu big data e people analytics. A combinação de people analytics com big data busca entender como base dados gigantescas podem servir de base para modelos de $\mathrm{hr}$ analytics preditivos de RH e auxiliar na compreensão do comportamento profissional.

O sétimo cluster (laranja) reuniu information management que trata da relevância da gestão da informação na construção do people analytics.

\section{CONSIDERAÇÕES FINAIS}

O presente trabalho teve por objetivo mapear a produção científica internacional sobre $h r$ analytics, a partir de uma exploração de artigos publicados na base Scopus. Observou-se que a pesquisa no campo se encontra em desenvolvimento no cenário internacional, com a estabilização no volume de artigos publicados, mas com indícios que está em crescimento e aparentemente não existe predominância exponencial de produção de artigos e reviews pelos autores, instituições e periódicos.

Quanto ao número de publicações por países, os Estados Unidos da América lideram o ranking de publicações, seguidos de Índia, Holanda e Reino Unido. Dessa forma, pode-se inferir que nesses países se encontra a maior parte das instituições e autores que possuem pesquisas relacionadas à temática de People Analytics, Human Resources Analytics e HR Analytics. Destaca-se que o Brasil possui somente 1 publicação relacionado ao tema pesquisado.

Neste artigo observou-se o ressurgimento enfático de Dave Ulrich como autor associado às funcionalidades do people analytics, o que sinaliza o fortalecimento do instrumentalismo em gestão de pessoas, sob a ótica do gerencialismo (managerialism). Esse aspecto do people analytics pode ensejar uma conotação reducionista dos sentidos de Gestão de Pessoas em 
termos de políticas e práticas, propiciando análises críticas de sua utilização nas organizações.

Encontrou-se ainda uma grande dispersão das publicações no que se refere aos autores, sendo que o principal autor (Dave Ulrich) representa menos de $2 \%$ do total das publicações pesquisadas. A University of Southern California foi encontrada como a maior fonte das publicações pesquisadas e o periódico Human Resource Management International Digest o maior produtor de documentos da amostra, representando $10 \%$ (5 documentos) do total da amostra.

Por meio de algoritmos de clusterização, verificou-se que a rede de coautoria, limitada a autores com pelo menos dois documentos na amostra, constituída de 9 clusters de colaboração em pesquisa, o que evidencia afluência inicial do campo. Entre estes agrupamentos, os que possuem mais elementos são justamente em torno dos autores mais prolíficos da amostra.

A rede de co-citação foi organizada em seis clusters: o primeiro formado por estudos que investigam a perspectiva que as organizações devem compreender em conjunto o procedimento operacional de people analytics quanto ao desenvolvimento estratégico de metodologias de recursos humanos e a consolidação do conceito de HR analytics. O segundo cluster aborda pesquisas dos processos de analytics com um diferencial para tomada de decisões mais conscientes. O terceiro cluster toma como base a caracterização deste grupo são predominantemente direcionadas à sobre a potencialidade e agregação de valor do $h r$ analytics, estudo de modelos de people analytics, relação entre processos de recursos humanos e a incorporação de tecnologias. Já o quarto cluster é formado por caracterização direcionado ao suporte da tecnologia da informação nos processos de recurso humanos. O quinto cluster são estudos realizados por estes pesquisadores focam sobre a influência da gestão de recursos humanos no desempenho organizacional com auxílio do people analytics. 0 sexto cluster tem como aspectos o foco a necessidade de mão de obra qualificada para manusear a tecnologia da informação relacionado a people analytics. 
Por fim, a rede de co-ocorrências de palavras-chave mostrou sete principais linhas de pesquisa sendo conduzidas: este conjunto sugere pesquisas que abordam as relações entre o people analytics e a gestão de talentos, à luz dos processos de retenção de talentos como estratégia em recursos humanos; Tais ocorrências permitem concluir como estudos estão considerando o $\mathrm{hr}$ analytics como uma mudança de gestão e sendo necessário para estratégia e agregando valor para os recursos humanos; como as pesquisas estão sendo desenvolvidas para tentar predizer os comportamentos humanos na gestão de recursos humanos; conjunto tenta compreender o comportamento humano e profissional de profissionais da área de anestesiologia através de métricas utilizadas em $h r$ analytics; 0 grupo tenta compreender o cenário do comércio e marketing, além de como as métricas de recursos humanos podem impulsionar a organização, principalmente nestes setores; busca entender como base dados gigantescas podem servir de base para modelos de $h r$ analytics preditivos de RH e auxiliar na compreensão do comportamento profissional e trata da relevância da gestão da informação na construção do people analytics.

Os dados apresentados permitiram verificar uma grande dispersão nos estudos sobre o tema, no entanto não foram encontrados grandes clusters de estudos, seja de autores ou instituições, ou uma área preponderante. É importante notar que o tema está em evolução no cenário mundial.

As temáticas encontradas nesta pesquisa contribuem para a disseminação do saber do assunto em investigação, pois vislumbra de maneira geral o cenário internacional, o que é people analytics, o que ela proporciona para ser aprofundada e posteriormente publicada no cenário acadêmico. Temáticas estas dão um respaldo teórico para várias pesquisas, auxiliando para ampliar estudo sobre o assunto analisado, provocando a posteriori para difusão do conhecimento do tema em questão.

Conclui-se, de maneira geral, que o tema people analytics encontrase em desenvolvimento na academia, existindo possibilidade de fomentá-lo ainda mais, mediante uma maior disseminação e socialização de novos autores, resultando a posteriori no surgimento de novos grupos de estudos; 
auxiliando para uma maior diversificação do tema na academia internacional e uma maior agregação e interação de autores sênior com os novos pesquisadores; e gerando mais influência numa maior densidade das redes de coautoria e das redes institucionais.

\section{REFERÊNCIAS}

Angrave, D., Charlwood, A., Kirkpatrick, I., Lawrence, M., \& Stuart, M. (2016). HR and analytics: why HR is set to fail the big data challenge. Human Resource Management Journal, 26(1), 1-11. doi: 10.1111/1748-8583.12090

Araújo, C. A. (2006). Bibliometria: evolução histórica e questões atuais. Em questão, 12(1), 11-32.

Bar-Ilan, J. (2008). Which h-index? A comparison of WoS, Scopus and Google Scholar. Scientometrics, 74(2), 257-271. doi: 10.1007/s11192-008-0216-y

Boudreau, J. (2017). HR Must Make People Analytics More User-Friendly. 2017.

Churchill Jr., G. A (1987). Marketing research: methodological foundations. Chicago: The Dryden Press, 1987

Egghe, L. (2005). Zipfian and lotkaian continuous concentration theory. Journal of the American Society for Information Science and Technology, 56(9), 935-945. doi: $10.1002 /$ asi.20186

Fitz-Enz, Jac; Mattox II, John (2014). Predictive analytics for human resources.

Frezatti, F.; Bido, De S. D., Capuano, C. P. A. \& Machado De C. J. M. (2015). A Estrutura de artefatos de controle gerencial no processo de inovação: existe associação com o perfil estratégico? Brazilian Business Review, 12(1), 129-156. doi: $10.15728 /$ bbr.2015.12.6

Gelbard, R., Ramon-Gonen, R., Carmeli, A., Bittmann, R. M., \& Talyansky, R. (2018). Sentiment analysis in organizational work: Towards an ontology of people analytics. Expert Systems, 35, e12289. doi: 10.1111/exsy.12289

Gülsoy, A.T. (2013). Human resource practices of an emerging-market multinational: Implications for enhancing organizational innovation. Procedia: Social and Behavioral Sciences, 75 (3):498-507. doi: 10.1016/j.sbspro.2013.04.054

Kessler, M. M. (1963). Bibliographic coupling between scientific papers. American Documentation, 14 (1), 10-25. doi: 10.1002/asi.5090140103

King, K. G. (2016). Data analytics in human resources: A case study and critical review. Human Resource Development Review, 15(4), 487-495. doi: $10.1177 / 1534484316675818$ 
Kovàcs, M. J. (2002). Morte e desenvolvimento humano. 4 ed. São Paulo: Casa do Psicólogo.

Leonardi, P., \& Contractor, N. (2018). Better PEOPLE Analytics Measure Who THEY KNOW, Not Just Who THEY ARE. HARVARD BUSINESS REVIEW, 96(6), 70-81.

Macedo, M. A. S., Nova, S. P. C. C., \& Almeida, K. (2009). Mapeamento e análise bibliométrica da utilização da Análise Envoltória de Dados (DEA) em estudos em contabilidade e administração. Contabilidade, Gestão e Governança, 12(3), 87101.

Macias-Chapula, C. A (1998). O papel da informetria e da cienciometria e sua perspectiva nacional e internacional. Ciência da Informação, Brasília, v. 27, n. 2, p. 134-140, maio/ago. doi: 10.1590/S0100-19651998000200005

Maia, E. L. S. (1973). Comportamento bibliométrico da língua portuguesa, como veículo de representação da informação. Ciência da Informação, 2(2).

Marler, J. H., \& Boudreau, J. W. (2017). An evidence-based review of HR Analytics. The International Journal of Human Resource Management, 28(1), 3-26. doi: $10.1080 / 09585192.2016 .1244699$

Moraes, M., Furtado, R. L., \& Tomaél, M. I. (2015). Redes de Citação: estudo de rede de pesquisadores a partir da competência em informação. Em Questão, 21(2), 181-202. doi: 10.19132/1808-5245212.181-202

Oliveira, S. C. M.; et al. Bibliometria em artigos de contabilidade aplicada ao setor público. In: CONGRES-SO BRASILEIRO DE CUSTOS, 20., 2013, Uberlândia. Anais.... São Leopoldo: Associação Brasileira de Custos, 2013.

Perianes-Rodriguez, A., Waltman, L., \& van Eck, N. J. (2016). Constructing bibliometric networks: A comparison between full and fractional counting. Journal of Informetrics, 10(4), 1178-1195. doi: 10.1016/j.joi.2016.10.006

Rasmussen, T., \& Ulrich, D. (2015). Learning from practice: how HR analytics avoids being a management fad. Organizational Dynamics, 44(3), 236-242. doi: $10.1016 /$ j.orgdyn.2015.05.008

Rostaing, H. (1997). La bibliométrie et sés techniques. Toulouse: Sciences de la Société.

Rocha, M. B.; Hoffmann, W. A. M. (2014). A produção científica brasileira sobre gestão do conhecimento na base de dados Web of Science. 2014. In: CONGRESSO BRASILEIRO DE GESTÃO DO CONHECIMENTO, 2014, Florianópolis. Anais... Florianópolis: SBGC, 2014

Silva, M. R. (2004). Análise bibliométrica da produção científica docente do programa de pós-graduação em educação especial/UFSCar (Dissertação de mestrado). Universidade Federal de São Carlos, São Carlos.

Singer, L. et al (2015). People Analytics in Software Development. In: International Summer School on Generative and Transformational Techniques in Software Engineering. Springer, Cham. p. 124-153. doi: 10.1007/978-3-319-60074-1_6 
Small, H. (1973). Co-citation in the scientific literature: a new measure of the relationship between two documents. Journal of the American Society for Information Science1, 24(4), 265-269. doi: 10.1002/asi.4630240406

Tursunbayeva, A., Di Lauro, S., \& Pagliari, C. (2018). People analytics-A scoping review of conceptual boundaries and value propositions. International Journal of Information Management, 43, 224-247. doi: 10.1016/j.ijinfomgt.2018.08.002

Ulrich, D., \& Dulebohn, J.H., Are we there yet? What's next for HR?, Human Resource Management Review (2015). doi: 10.1016/j.hrmr.2015.01.004

van Eck, N. J., \& Waltman, L. (2014). Visualizing bibliometric networks. In Measuring scholarly impact (pp. 285-320). Springer, Cham.

Vargas, R., Yurova, Y. V., Ruppel, C. P., Tworoger, L. C., \& Greenwood, R. (2018). Individual adoption of HR analytics: a fine grained view of the early stages leading to adoption. The International Journal of Human Resource Management, 1-22. doi: $10.1080 / 09585192.2018 .1446181$

Zupic, I., \& Čater, T. (2015). Bibliometric methods in management and organization. Organizational Research Methods, 18(3), 429-472. doi: $10.1177 / 1094428114562629$ 